\title{
Epidemiology of Kaposi's sarcoma in Zambia, 2007 - 2014
}

\author{
Maybin Kalubula $^{1,2^{*}}$, Heqing Shen ${ }^{1,2^{*}}$, Mpundu Makasa ${ }^{3}$
}

1. Key Lab of Urban Environment and Health, Institute of Urban Environment, Chinese Academy of Sciences, Xiamen 361021, China

2. University of Chinese Academy of Sciences, Beijing 100049, China;

3. The University of Zambia, School of Medicine, Ridgeway Campus, P. O. Box 50110 Lusaka, Zambia.

Correspondance: Maybin Kalubula; mkalubula@gmail.com

\section{Background}

Kaposi's sarcoma (KS) is the most prevalent HIV and AIDS-associated cancer in the world. Zambia has been considered as part of the "KS belt", where endemic KS has been prevalent. This study, therefore, aimed to present the descriptive epidemiology of Kaposi's sarcoma in Zambia from 2007 - 2014.

Methods

We conducted the descriptive epidemiology of Kaposi's sarcoma in Zambia nested on two data sources; the Zambia National Cancer Registry (ZNCR) Kaposi's sarcoma (KS) data, and population-based HIV data from the Zambia National AIDS Council (NAC). Central Statistics Office (CSO) demographic data were used to determine the prevalence and annual incidence of KS. KS sample was 2521 while HIV data from NAC were already population-based (HIV impact assessment survey). We used Microsoft Excel and SPSS version 21 in graphical computation and statistical analyses.

Results

Both HIV and KS were highly prevalent in Lusaka, Central, and Southern provinces. ART coverage ranged from 40\% - 60\%; HIV prevalence was $14.9 \%$ in females and $9.5 \%$ in males while KS prevalence was 13/100,000 in females and 21/100,000 in males. HIV prevalence was associated with KS prevalence with $\mathrm{r}=0.827$ and a p-value of 0.001 in males, and $\mathrm{r}=0.898$ with a $\mathrm{p}$-value of 0.000 in females. There were $61 \%$ confirmed HIV seropositive KS, $18 \%$ confirmed HIV seronegative KS and $21 \%$ unknown HIV status KS. Conclusions

The high prevalence of KS in Zambia is as a result of the high prevalence of HIV. The identified two key interventions for the reduction of KS morbidity are; reducing HIV infection rate and improving ART coverage across the country.

Keywords: Epidemiology of Kaposi's sarcoma; HIV- Kaposi's sarcoma; Kaposi's sarcoma in Zambia; HIV prevalence in Zambia

\section{Introduction}

The most prevalent HIV and AIDS-associated cancer in the world is Kaposi's sarcoma (KS). It mostly affects the skin although internal visceral organs are also affected ${ }^{1}$. Moritz Kaposi first described KS in 1842 and has since been classified into four clinical-epidemiological variants namely; the "original classic," endemic or African KS, iatrogenic or immunosuppression-related KS, and epidemic or AIDSrelated $\mathrm{KS}^{2,3}$. The epidemiology of these $\mathrm{KS}$ variants varies considerably around the globe. For instance, the "original classic" variant of KS has been observed mostly in elderly men of Mediterranean or eastern European origin while the classic variant of $\mathrm{KS}$ generally affects more men than women ${ }^{4,5}$. AIDS-associated KS is different from other KS variants because of its aggressiveness manifested in immunocompromised patients by way of targeting mucosal tissues before advancing to visceral organs leading to organ dysfunction and death ${ }^{6-8}$.

Epidemiological studies have indicated that the AIDS epidemic in Africa has resulted in an increased incidence of KS. Notable differences were observed from 1968 to 1970 when KS accounted for $6.6 \%$ of all cancers occurring in men which rose to $48.6 \%$ during the 1989 to 1991 period following the AIDS epidemic ${ }^{9,10}$. The impact of HIV/ AIDS in Sub Saharan Africa brought about an increase in the percentage of KSHV positive children aged four to nine years to approximately $70 \%$. Almost all these children were born HIV positive which they acquired from their infected

\section{mothers (mother to child transmission) ${ }^{11}$.}

The status of the HIV epidemic in Zambia dates back to 1984 when the first case of AIDS was diagnosed ${ }^{12}$. Since then HIV has spread throughout the Zambian population with HIV prevalence peak in the 1990s which was estimated at $14.3 \%$ in the year 2007 with a higher prevalence in women (16.1\%) compared to $12.3 \%$ in men ${ }^{12}$. The impact of HIV/ AIDS in Zambia has prompted the Zambian Government and cooperating partners to embark on ART services as a way of reducing HIV related morbidity and mortality in the country.

ART services in Zambia started during the early 2000s with a notable increase in ART coverage during 2002 to 2003 period $^{13}$. From the time of ART inception in Zambia, HIV positive patients with CD4 count less than 200 or $250-350$ for pregnant women were eligible to be initiated on ART. From August 2017, the Zambian Government adopted WHO guidelines for the management of HIV patients and instituted a mandatory HIV testing policy for anyone seeking medical services in public hospitals. Additionally, ART was initiated to all HIV positive individuals regardless of their CD4 count.

The emergence of HIV/ AIDS in Zambia in the early 1980s corresponded with the increase in both endemic and HIV related KS incidences and has since been considered part of the "Kaposi's sarcoma belt"14. Furthermore, children infected with HIV had a five-fold higher risk of acquiring KSHV compared with HIV uninfected children in Zambia ${ }^{15}$. 
As such, the government embarked on the prevention of mother to child transmission (PMTCT) program which has the potential to reduce the risk of HIV and KSHV transmission from infected mothers to children ${ }^{16}$. The aim of this study was to present the descriptive epidemiology of Kaposi's sarcoma in Zambia from 2007 - 2014.

\section{Materials and methods}

\section{Study site}

The Republic of Zambia is a landlocked country located in the southern part of the African Continent. It has eight neighbouring countries namely, Tanzania to the north, Malawi to the east, Mozambique to the south-east, Zimbabwe to the south, Botswana to the south-west, Namibia to the south-west, Angola to the west and Democratic Republic of Congo (DRC) to the north-west. Zambia lies between latitudes $8^{\circ}$ and $18^{\circ}$ south, and longitudes $22^{\circ}$ and $34^{\circ}$ east, and has a geographical area of $753,612 \mathrm{~km}^{217}$.

\section{Study design}

We conducted a descriptive study of Kaposi's sarcoma (KS) in Zambia nested on two data sources; the Zambia National Cancer Registry (ZNCR) Kaposi's sarcoma data, and population-based HIV data from the Zambia National AIDS Council (NAC) from 2007 to 2014. ZNCR collects and keeps population-based cancer data in Zambia with major sources of data being Health Management Information System (HMIS), Private and Government Hospital Registries which are also linked from grass root level by health centres and communities. Other sources include Community Health Workers (CHW), death records and Churches Health Organization (CHAZ). Zambia National Cancer Registry has standard registers that are distributed and used in all health facilities countrywide. ZNCR has been transitioning into a Population-based Registry with Lusaka having attained that status. All captured cancer cases are reported to ZNCR and are coded with ICD10, Primary Site, and Histology codes among others. Histology Laboratory investigation accounts for $70 \%$ of cancer diagnoses while surgery, chemotherapy, and radiology are the three major treatment modalities in Zambia. The cancer register is comprehensive and captures the cause of death as well as HIV status of patients among other indicators. All reported cases are then entered in the ZNCR database. ZNCR conducts regular data audits to ensure that the collected data is of quality.

Zambia National AIDS Council works hand in hand with the Ministry of Health of The Republic of Zambia. NAC is concerned with HIV/AIDS/STI and TB programs in Zambia and keeps information pertaining to HIV/AIDS/STI and TB in Zambia. The Ministry of Health and National AIDS Council conducted a population-based HIV Impact Assessment which was finalized in 2016. We were availed with HIV data which we used together with KS data in this study. We also obtained population data from the Central Statistics Office (CSO) which was used to determine KS prevalence and annual incidence rates.

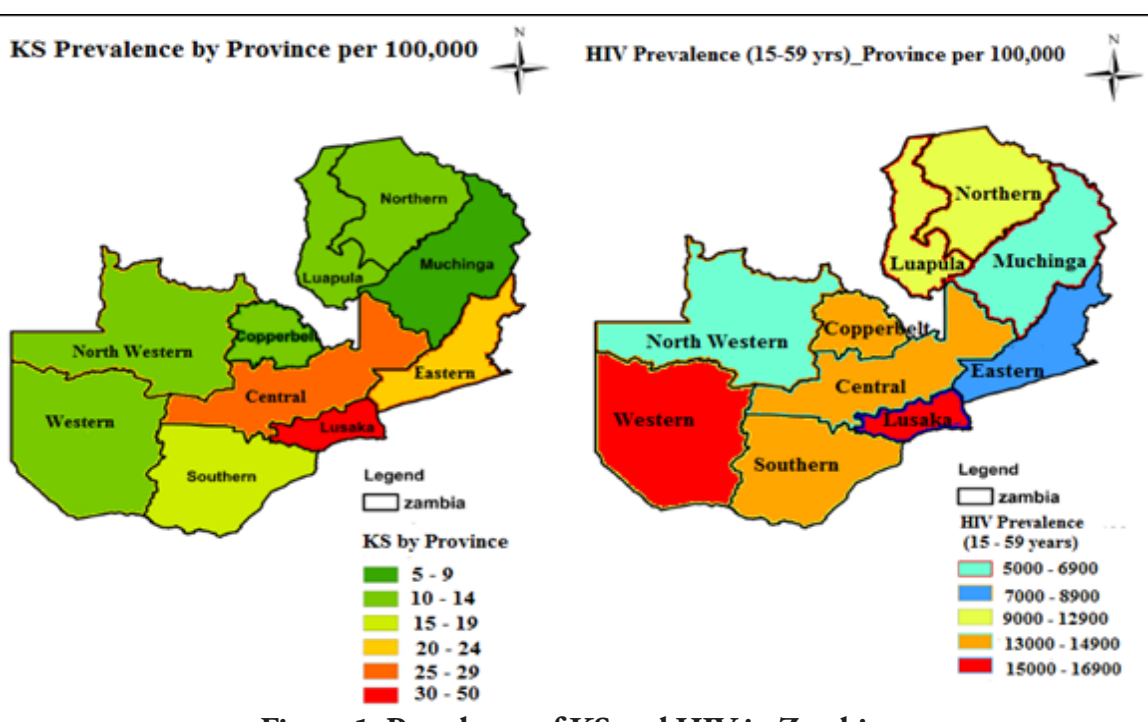

Figure 1: Prevalence of KS and HIV in Zambia at the provincial level was done using Geographical Information System (ArcGIS) version 10.3.1, Redlands, CA. This study was approved by the Zambia National Health Research Authority and the University of Zambia Biomedical Research Ethics Committee (UNZABREC) as well as the Research Ethics Committee of the Institute of Urban Environment, Chinese Academy of Sciences.

\section{Sampling}

Kaposi's sarcoma is classified under non-melanoma skin cancers with ICD10 code C460. It is coded 9140 in the ZNCR database. We were able to filter through the system records using this code and bearing in mind the study period from 2007 to 2014. KS sample was $2521(\mathrm{n}=2521)$ in this study.

HIV data were obtained from a nationally representative two-stage stratified cluster sample design, where the first stage selected 511 enumeration areas from the 2010 Zambia Census of Population and Housing, using a probability proportional method. The second stage randomly selected a sample of households in each enumeration area known as a cluster using an equal probability method, where the average number of households selected per cluster was 27 and the actual number of households selected per cluster ranged from 11 to $48^{18}$. Therefore, HIV data from NAC were population-based and did not any require further synthesis using CSO demographic data in the current study.

\section{Data Analyses}

Prevalence and incidence rates were determined using the Zambia central statistics office provincial populations. SPSS version 21 regression model was used to determine the association between Kaposi's sarcoma and HIV prevalence across age groups and Pearson Correlation was used to determine the correlation coefficient. Microsoft Excel was used for graphical computation and analysis of KS and HIV prevalence by age group. In addition to this, we linked all patients' data to provinces of origin using their residential addresses and then mapped the distribution of both KS and HIV using GIS (ArcGIS) version 10.3.1, Redlands, CA.

\section{Results}

Concerning GIS mapping, we linked all patients' data to provinces of origin using their residential addresses. Mapping of the distribution of Kaposi's sarcoma and HIV prevalence 
Prevalence of Kaposi's sarcoma and HIV by the province in Zambia

Figure 1 shows the geographical distribution of Kaposi's sarcoma and HIV prevalence in people aged 15-59 years by province in Zambia. Kaposi's sarcoma was widely distributed in all the ten provinces although Lusaka, Central and Eastern provinces were the most affected with prevalence rates of 48 , 25 and 20 per 100,000 population respectively. Furthermore, the northern zone comprising Muchinga, Northern, Luapula, Copperbelt and North-Western provinces generally had low prevalence of Kaposi's sarcoma while the southern zone comprising Eastern, Lusaka, Central, Western and Southern provinces registered more cases of KS.

Throughout the study period, we did not observe any association between HIV prevalence and KS prevalence at provincial level. In addition, HIV prevalence among people aged 15 to 59 years was highest in Lusaka Province with the rate of 16,100 per 100,000 population followed by Western $(16,000)$, Copperbelt $(14,200)$, Central and Southern provinces $(13,400)$ while other provinces had relatively low HIV prevalence. Table 1 shows the details.

Table 1: Prevalence of Kaposi's sarcoma and HIV in Zambia

\begin{tabular}{lcccc}
\hline \multicolumn{5}{c}{ Prevalence per 100,000 population } \\
Province & Male & Female & Total & HIV in adults \\
aged 15 - 59 years \\
\hline Central & 27.7 & 22.1 & 25 & 13400 \\
Copperbelt & 10.2 & 9.2 & 10 & 14200 \\
Eastern & 25.2 & 14.1 & 20 & 8200 \\
Luapula & 15.2 & 10.2 & 13 & 9300 \\
Lusaka & 56.9 & 39.1 & 48 & 16100 \\
Muchinga & 9.2 & 8.9 & 9 & 5900 \\
Northern & 13.2 & 10.2 & 12 & 9700 \\
North & 15.1 & 8.6 & 12 & 6900 \\
Western & & & & \\
Southern & 17.6 & 12.1 & 15 & 13400 \\
Western & 14.7 & 9.7 & 12 & 16000 \\
\hline
\end{tabular}

HIV and Kaposi's sarcoma prevalence by age and sex in Zambia

The HIV prevalence in Zambia was 12,300 per 100,000 population and was significantly higher among females $(14,900)$ compared to males $(9,500)$ per 100,000 population during the study period. By age group and sex, HIV prevalence was highest among females in the age group $40-$ 44 , followed by the $45-49$, and $35-39$ age groups in that order. In males, age groups $40-44,45-49$ and $50-54$ were the most HIV prevalent. Figure 2 shows details of HIV prevalence by age group and sex in Zambia.

Kaposi's sarcoma prevalence by sex in Zambia was observed to be the opposite of HIV prevalence. Kaposi's sarcoma was more prevalent in males (21 per 100,000) compared to females $(13$ per 100,000) in Zambia. At the national level, KS prevalence was 17 per 100,000 population. By age group and sex, KS prevalence was highest in people aged 80 years and above in both sexes. High prevalence was also noticeable in the $30-34,35-39,40-44$, and $45-49$ age groups for both males and females. Figure 3 shows the details of KS prevalence by age group and sex in Zambia.
Association between $H I V$ prevalence and $K S$ prevalence by sex and age

KS prevalence was associated with HIV prevalence across age groups and sex. In males, HIV prevalence was strongly associated with KS prevalence with correlation coefficient $=0.827$ and $\mathrm{p}$-value of 0.001. In females, HIV prevalence had a strong association with KS prevalence with correlation coefficient $=0.898$ and $p$-value of 0.000 . This suggests that the risk of getting KS increases with HIV for any particular age group.



Figure 2: HIV prevalence in people aged 0 - 59 years by age group and sex in Zambia

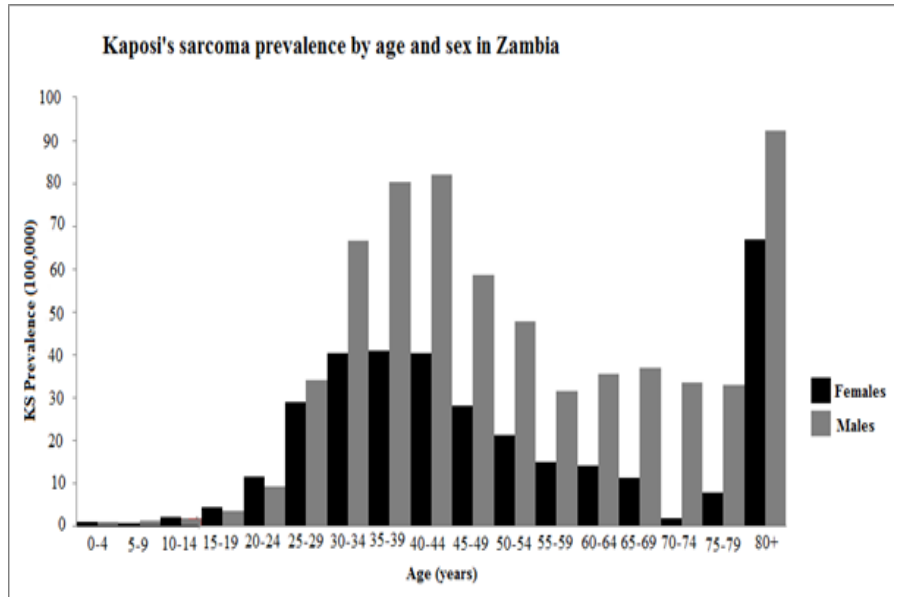

Figure 3: Kaposi's sarcoma prevalence by age group and sex in Zambia

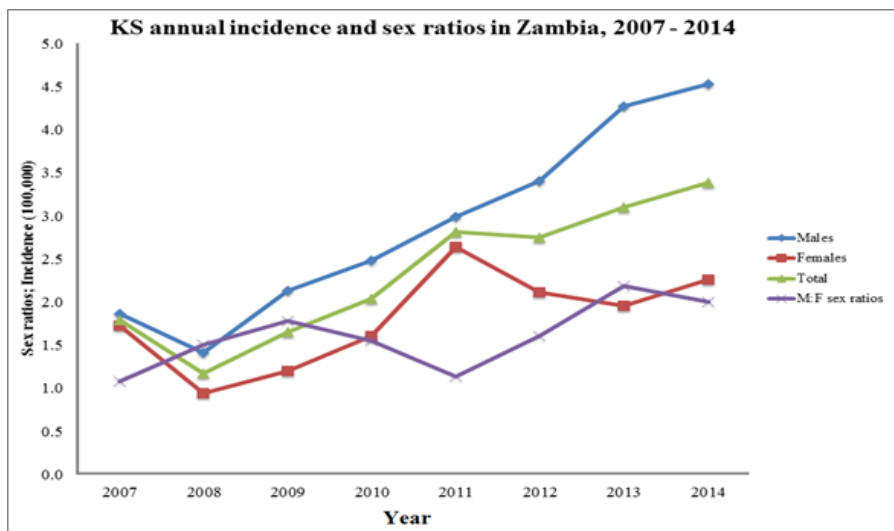

Figure 4: Kaposi's sarcoma incidence and sex ratios in Zambia 
Distribution of HIV status among Kaposi sarcoma cases in the Zambia Cancer Registry

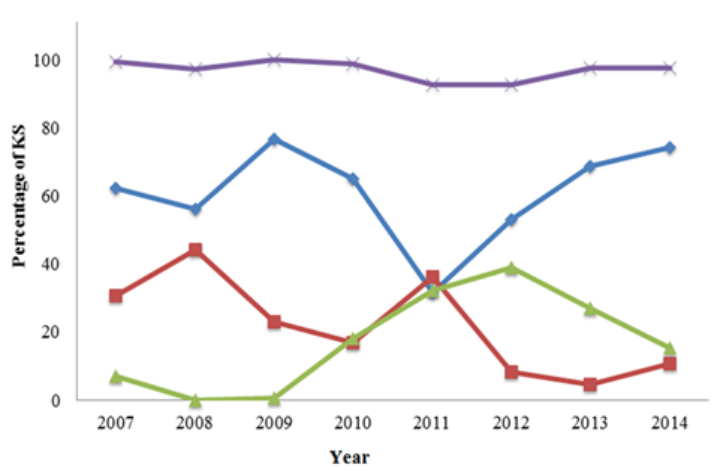

Figure 5: Distribution of HIV status among Kaposi sarcoma cases

\section{Trends of Kaposi's sarcoma incidence by sex in Zambia, 2007- 2014}

Kaposi's sarcoma incidence in Zambia showed an upward trend from the baseline year 2007 to the year 2014. This means that the burden of KS in Zambia continued to be a major health problem throughout the entire period. Figure 4 shows the trends of KS annual incidence by sex and sex ratios in Zambia. M: F sex ratios were greater than one throughout the study period, an indication that KS incidence was significantly higher among males compared to females during the study period. Note that M: F sex ratios are absolute numbers while KS annual incidence rates are per 100,000 population.

\section{Trends of HIV KS, HIV seronegative KS and HIV KS on ART in Zambia, 2007 - 2014}

Among all KS cases, 61\% were confirmed HIV seropositive $\mathrm{KS}, 18 \%$ confirmed HIV seronegative KS, and $21 \%$ had unknown HIV status in Zambia. These results indicate that $\mathrm{HIV}$ testing coverage among KS patients was $79 \%$ during the study period. If $21 \%$ of unknown HIV status were tested, the percentage of HIV KS could be much higher than $61 \%$. $\mathrm{HIV}$ is a major risk factor for KS in Zambia. There were 59\% of KS patients on ART during the study period. This means that $2 \%$ of HIV KS was not initiated on ART. Taking HIV seropositive KS as a denominator, HIV KS ART coverage was $97 \%$.

There were fluctuations in the annual trends of HIV seropositive KS, HIV seronegative KS, and unknown HIV status KS while HIV KS ART coverage was above $90 \%$ throughout the study period. HIV KS ART coverage was far much higher than ART coverage in the general Zambian population which ranged from $40 \%$ to $60 \%$ during the study period. The observed increase in KS was as a result of low ART coverage in the general population. HIV testing among KS patients was good from 2007 to 2009 and became poor from 2009 onward as reflected by the trend of unknown HIV status KS. Figure 5 shows trends of HIV seropositive KS, HIV seronegative KS, unknown HIV status KS and HIV KS on ART from 2007 to 2014.

\section{Discussion}

This study has shown that both Kaposi's sarcoma and $\mathrm{HIV}$ are widely distributed in Zambia although notable geographical variations were observed at the provincial level. Kaposi's sarcoma was more prevalent in Eastern, Lusaka, Central and Southern provinces while HIV was more prevalent in Lusaka, Western, Copperbelt, Central and Southern provinces. Although there was no statistically significant association between HIV prevalence and KS prevalence in terms of geographical distribution, this study observed that Lusaka, Central and Southern provinces had both high HIV prevalence and high KS prevalence.

On the other hand, our study established a strong association between HIV prevalence and KS prevalence across age stoups in both males and females. This strong association indicates that HIV is a major risk factor for Kaposi's sarcoma in Zambia. These results are similar to the results of previous studies ${ }^{19-23}$ which indicated a strong association between HIV and $\mathrm{KS}$.

By gender, KS was more prevalent in males than females across all age groups. This entails that gender is a risk factor for KS. These results are similar to a study conducted in Uganda where Kaposi's sarcoma preferentially affected more males than females ${ }^{22}$. Furthermore, KS has a tendency to incline towards certain geographical regions and affects some races more than others ${ }^{22}$.

This study has observed an upward trend of Kaposi's sarcoma incidence in Zambia from the baseline year 2007 to the year 2014. This confirms that KS continues to be a major health problem in Zambia. The emergence of HIV-1 epidemic in Zambia during the early 1980s has resulted in the significant increase in $\mathrm{KS}$ incidence in the later decades indicated by the $\mathrm{HIV}$ and KS association. Zambia is considered as part of the "KS belt" due to the significant increase in KS incidence following the emergence of the HIV-1 epidemic ${ }^{14,24-26}$.

Our study observed that HIV KS prevalence was 61\% compared to $18 \%$ HIV negative $\mathrm{KS}$ in Zambia during the study period. Low HIV testing coverage left $21 \%$ of KS patients with unknown HIV status. There is a need to increase HIV testing coverage not only among KS patients but also the general Zambian population as this would be helpful in the confirmation of HIV status as well as early initiation of $\mathrm{HIV}$ patients on ART thereby reducing KS incidence. This study established that ART coverage among HIV seropositive KS patients (97\%) was far much higher than ART coverage in the general Zambia population $(60 \%)$. This variation in ART coverage is attributed to differences in health-seeking behavior between HIV seropositive KS patients and HIV patients in general. HIV seropositive KS patients are compelled to test and be initiated on ART during cancer diagnosis and treatment process while most HIV patients do not seek medical attention in good time until their CD4 count drastically drop.

Our study discovered that KS patients in the age groups 20 $-29,30-39$, and $40-49$ years were the most affected. Notably, the emergence of HIV-1 in Zambia during the 1980 s as corresponded with the increased prevalence of KS 24-26. Furthermore, the limited health education regarding HIV spread and prevention such as the use of condoms made useful and sexually active people very vulnerable to HIV-1 virus infection. The third factor is that the health delivery system in Zambia was not up-to-date hence did not adequately provide the much-needed ART services. Most KS cases emerge from immunocompromised and HIV positive patients ${ }^{27-31}$ which have also been confirmed by this study as 61\% of KS were HIV seropositive.

The two key intervention points for the reduction of KS morbidity are: the HIV infection point (reduced HIV infection results in reduced HIV morbidity hence reduced KS since the two have a strong association) and improving ART services and coverage. The two key interventions https://doi.org/10.4314/mmj.v32i2.4 
should be implemented at a higher level to prevent current and future generations from KS-HIV scourge. Furthermore, health workers in Zambia should be oriented in active cancer screening including those manning rural health centers. A similar approach worked in high-income countries following the advent of combined antiretroviral therapy, the prevalence of epidemic KS has dramatically decreased ${ }^{32}$.

KS prevalence has been high not only in Zambia but also Southern Africa as a whole due to high prevalence of HIV in the region. Zimbabwe and South Africa have a significantly high prevalence of KS. According to a study conducted in South Africa, the incidence of KS was substantially lower among patients on ART than those not on ART. Early ART initiation and prevention of immune suppression significantly reduce the risk of KSHV acquisition among HIV-infected children in an area where both viruses are highly endemic. This means that timely initiation of ART is essential to prevent $\mathrm{KS}$ and $\mathrm{KS}$-associated morbidity and mortality in regions with high burden of $\mathrm{HIV}^{33}$. In the same vein, the Zambian Government adopted WHO guidelines for the management of HIV patients in August 2017. HIV testing became mandatory in Zambia implying that anyone who seeks medical attention at the health facility has to be tested. Those found HIV positive are initiated on ART regardless of their CD4 count in accordance with WHO guidelines. This approach would result in the reduction of HIV-related KS over a period of time ${ }^{34}$.

This study had some limitations; it could not assess other confounding KS risk factors apart from HIV and gender because the initial aim of data capturing by ZNCR was not in line with our research objectives. Our study focused on KS from 2007 to 2014 and could not assess the current trends of KS after the Zambian Government adopted WHO guidelines for mandatory HIV testing and ART initiation in 2017. There is need for another study to assess KS epidemiology in Zambia after the adoption of WHO guidelines.

In conclusion, the high prevalence of KS in Zambia is as a result of high HIV prevalence. The identified two key interventions for the reduction of KS morbidity are: reducing HIV infection and improving ART coverage from the current $60 \%$ to above $90 \%$ across the country. Health institutions both rural and urban should be strengthened for early cancer detection and to intensify health education in order to curb the scourge.

\section{Competing Interests}

Authors declare that they have no competing interests.

\section{Authors' Contributions}

MK designed the study, developed and programmed the model and drafted the manuscript. MM conceived the study. HS conceived the study and approved the model. All authors read and approved the manuscript.

\section{Acknowledgements}

Compliments go to the National Health Research Authority of the Republic of Zambia, University of Zambia Biomedical Research Ethics Committee and the Research Ethics Committee of the Institute of Urban Environment, Chinese Academy of Sciences for allowing the researchers to conduct a study in Zambia. The study was supported by the Institute of Urban Environment in collaboration with the University of Chinese Academy of Sciences (UCAS).

\section{References}

1. Liu z, Fang Q, Zuo J, et al. The worldwide incidence of Kaposi's sarcoma in the HIV/AIDS era. HIV Med. 2018 May;19(5):355-364. doi: 10.1111/hiv.12584.

2. Orem J. Cancer prevention and control: Kaposi's sarcoma. Ecancermedicalscience. 2019; 13: 951. doi: 10.3332/ecancer.2019.951

3. Dourmishev L, Dourmishev A, Palmeri D, Schwartz R, Lukac D. Molecular genetics of Kaposi's sarcoma-associated herpesvirus (human herpesvirus 8) epidemiology and pathogenesis. Microbiol Mol Biol Rev, 2003; 63: 175-212.

4. Curtiss P. Strazulla LC, Friedman-Kien AE. An Update on Kaposi's Sarcoma: Epidemiology, Pathogenesis and Treatment. Dermatol Ther (Heidelb). 2016; 6(4): 465-470.

5. Dourmishev LA, et al. Molecular genetics of Kaposi's sarcomaassociated herpesvirus (human herpesvirus 8) epidemiology and pathogenesis. Microbiol Mol Biol Rev. 2003;67(2):175-212.

6. Koski L, Ngoma T, Mwaiselage J, Le L, Soliman AS. Changes in the pattern of Kaposi's sarcoma at Ocean Road Cancer Institute in Tanzania (2006-2011). Int J STD AIDS. 2015;26(7):470-8. doi: $10.1177 / 0956462414544724$.

7. Hengge UR, et al. Update on Kaposi's sarcoma and other HHV8 associated diseases. Part 1: epidemiology, environmental predispositions, clinical manifestations, and therapy. Lancet Infect Dis. 2002;2(5):281-292.

8. Vicente S, Francisco M, Eduardo B, et al. Epidemiology and clinical characteristics of classic Kaposi's sarcoma, seroprevalence, and variants of human herpesvirus 8 in South America: A critical review of an old disease. International Journal of Infectious Diseases. 2005; 9: 239-250 https://doi.org/10.1016/j.ijid.2005.02.004

9. Sasco AJ, Jaquet A, Boidin E, et al.The Challenge of AIDS-Related Malignancies in Sub-Saharan Africa. Plos One. 2010. https://doi. org/10.1371/journal.pone.0008621

10. Ruocco E, Ruocco V,Tornesello ML, et al. Kaposi's sarcoma:Etiology and pathogenesis, inducing factors, causal associations, and treatments: Facts and controversies. Clinics in Dermatology.2013;31:413 - 422.

11. de Souza VA, Sumita LM, Nascimento MC, Oliveira J, Mascheretti M, Quiroga M, Freire W.S, Tateno A, Boulos M, Mayaud P, et al. Human Herpesvirus- 8 infection and oral shedding in Amerindian and non-Amerindian populations in the Brazilian amazon region. J. Infect. Dis. 2007 ; 196:844-852.

12. Zambia Ministry of Health, National AIDS Council. Global AIDS Response Progress Reporting (GARPR) Zambia Country Report 2013. Ministry of Health, Zambia. 2014; (13):2.

13. Zambia National HIV/AIDS/STI/TB Council. Zambia HIV Prevention Response and Modes of Transmission Analysis 2009 Final Report. Zambia National AIDS Council. 2009: 33.

14. Cesarman E, Damania B, Krown SE, et al. Kaposi Sarcoma. Nat Rev Dis Primers. 2019; 5(1): 9. doi: 10.1038/s41572-019-0060-9.

15. Minhas V, Crabtree KL, Chao A, et al. Early Childhood Infection by Human Herpesvirus 8 in Zambia and the Role of Human Immunodeficiency Virus Type 1 Coinfection in a Highly Endemic Area. Am J Epidemiol. 2008;168(3):311-320.

16. Olp LN, Minhas V, Gondwe C, Kankasa C, et al. Effects of Antiretroviral Therapy on Kaposi's Sarcoma-Associated Herpesvirus (KSHV) Transmission Among HIV-Infected Zambian Children. Journal of the National Cancer Institute. 2015 Oct;107(10).

17. Zambia latitude and longitude map. Source: http//www. mapsofworld.com/lat-_long/zZambialat-long.html.

18. Ministry of Health, Zambia. Zambia Population-based HIV Impact Assessment (ZAMPHIA) 2016: First Report. Zambia, Ministry of Health. December 2017; (1):10 
19. Ziegler J, Newton R, Bourboulia D, et al. Risk factors for Kaposi's sarcoma: A case-control study of HIV-seronegative people in Uganda. Int. J. Cancer. 2003; 103:233-240

20. Goedert JJ, Vitale F, Lauria C, Mario D, et al. Risk factor for Classical Kaposi's sarcoma. Journal of the National Cancer Institute. 2002;94 (22): 1712-1718.

21. Stolka K, Ndom P, Hemingway-Foday J, et al. Risk factors for Kaposi's sarcoma among HIV- positive individuals in a case-control study in Cameroon. Cancer Epidemiol. 2014; 38 (2): 137-143.

22. Phipps W, Ssewankambo F, Nguyen H, et al. Gender Differences in Clinical Presentation and Outcomes of Epidemic Kaposi Sarcoma in Uganda. PLoS One. 2010; 5(11): e13936. doi: 10.1371/journal. pone.0013936.

23. Lupia R, Wabuyia P.B, Ontio P, et al. Risk factors for Kaposi's sarcoma in human immunodeficiency virus patients after initiation of antiretroviral therapy: A nested case-control study in Kenya. Journal of Microbiology, Immunology and infection. 2017; 50 (6): 781-788.

24. Minhas V, Crabtree KL, Chao A, et al. The Zambia Children's KS-HHV8 Study: Rationale, Study Design, and Study Methods. Am J Epidemiol. 2011; 173(9): 1085-1092. doi: 10.1093/aje/kwq465

25. Minhas V, Wood C. Epidemiology and Transmission of Kaposi's Sarcoma-Associated Herpesvirus. Viruses. 2014; 6(11): 4178-4194

26. Kumar P, Kuwa NY, Minhas V, et al. Higher Levels of Neutralizing Antibodies against KSHV in KS Patients Compared to Asymptomatic Individuals from Zambia. Plos One. 2013. https://doi.org/10.1371/ journal.pone.0071254

27. Haverkos HW. Multifactorial etiology of Kaposi's sarcoma: a hypothesis. Journal of Biosciences. 2008 Dec;33(5):643-51.
28. Phipps W, Ssewankambo F, Nguyen H, Saracino M, Wald A, Corey L, et al. Gender differences in clinical presentation and outcomes of epidemic Kaposi sarcoma in Uganda. PloS one. 2010 Jan;5(11):e13936.

29. Yan S, Huang J, Zheng Q, Zhu H, et al. A rare case of an HIVseronegative AIDS patient with Pneumocystis jirovecii pneumonia. BMC Infectious Diseases. 2019: 525

30. Wang J, Reid H, Klimas N, Koshelev M. An unusual series of patients with Kaposi sarcoma. JAAD Case Rep. 2019 Aug; 5(8): 646649. doi: 10.1016/j.jdcr.2019.05.016

31. Silverberg MJ, Neuhaus J, Bower M, Gey D, Hatzakis A, Henry $\mathrm{K}$, et al. Risk of cancers during interrupted antiretroviral therapy in the SMART study. AIDS (London, England) 2007 Sep 12;21(14):1957-63.

32. Engels EA, Pfeiffer RM, Goedert JJ, Virgo P, McNeel TS, Scoppa $\mathrm{SM}$, et al. Trends in cancer risk among people with AIDS in the United States 1980-2002. AIDS (London, England) 2006 Aug;20(12):164554.

33. Bohlius J, Valeri F, Maskew M, et al. Kaposi's Sarcoma in HIVinfected Patients in South Africa: Multicohort Study in the Antiretroviral Therapy Era. Int J Cancer. 2014 Dec 1;135(11):2644-52. doi: 10.1002/ ijc. 28894

34. Olp LN, Mihas V, Gondwe C, et al. Effects of Antiretroviral Therapy on Kaposi's Sarcoma-Associated Herpesvirus (KSHV) Transmission Among HIV-Infected Zambian Children. JNCI: Journal of the National Cancer Institute, 2015 Oct; 107 (10). https://doi.org/10.1093/jnci/ djv189 